\title{
Macleaya cordata extracts exert antiviral effects in newborn mice with rotavirus-induced diarrhea via inhibiting the JAK2/STAT3 signaling pathway
}

\author{
CHUNMAO JIANG $^{1 *}$, HAIFENG YANG ${ }^{2 *}$, XIAOLAN CHEN ${ }^{2}$, SHULEI QIU ${ }^{1}$, \\ CAIHONG WU ${ }^{1}$, BIN ZHANG ${ }^{3}$ and LIQIN JIN ${ }^{2}$ \\ ${ }^{1}$ School of Veterinary Medicine; ${ }^{2}$ School of Animal Pharmaceutical Sciences; ${ }^{3}$ School of Pet Science and Technology, \\ Jiangsu Agri-Animal Husbandry Vocational College, Taizhou, Jiangsu 225300, P.R. China
}

Received April 4, 2019; Accepted January 16, 2020

DOI: $10.3892 /$ etm.2020.8766

\begin{abstract}
Accumulating evidence demonstrates that Macleaya cordata extract exerts antiviral and anti-inflammatory effects in various diseases. The present study aimed to investigate the potential effects of $M$. cordata on rotavirus SA11-induced diarrhea in mice. Diarrhea severity, levels of inflammatory cytokines, histological changes in the small intestine and the underlying mechanisms were evaluated in rotavirus-stimulated mice treated with 1,2 and $4 \mathrm{mg} / \mathrm{kg} /$ day $M$. cordata or $4 \mathrm{mg} / \mathrm{kg} /$ day ribavirin (positive control). $M$. cordata treatment effectively ameliorated rotavirus-induced diarrhea in a dose-dependent manner by decreasing viral RNA levels. In addition, $M$. cordata reduced the release of pro-inflammatory cytokines including migration inhibitory factor, interleukin (IL)-8, IL- $\beta$, interferon- $\gamma$ and tumor necrosis factor- $\alpha$, and elevated the secretion of the anti-inflammatory cytokine IL-10 following rotavirus infection. $M$. cordata inhibited intestinal epithelial cell apoptosis and improved intestinal inflammation after rotavirus infection. The study also revealed that $M$. cordata exerted antiviral and anti-inflammatory effects on rotavirus-induced diarrhea by suppressing the Janus kinase 2 (JAK2)/STAT3 pathway, as reflected by decreased protein expression of phosphorylated (p)-JAK2 and p-STAT3. Overall, $M$. cordata effectively inhibited the inflammation caused by rotavirus, which was closely associated with the suppression of JAK2/STAT3 phosphorylation. These data
\end{abstract}

Correspondence to: Professor Caihong Wu, School of Veterinary Medicine, Jiangsu Agri-Animal Husbandry Vocational College, 8 Feng Huang East Road, Taizhou, Jiangsu 225300, P.R. China

E-mail: caihongwu616@aliyun.com

*Contributed equally

Key words: rotavirus, diarrhea, Macleaya cordata, inflammatory cytokines, Janus kinase 2/STAT3 signaling pathway suggested that $M$. cordata may be applied as a treatment for rotavirus-induced diarrhea.

\section{Introduction}

Rotavirus is a double-stranded RNA virus which remains a primary etiologic agent of viral gastroenteritis and diarrhea in young children and animals (1). In humans, rotavirus is responsible for $\sim 450,000$ deaths worldwide per year in children under 5 years old (2). Rotavirus infection mainly occurs in the small intestine, which plays a role in food digestion and absorption (3). Current strategies to prevent rotavirus transmission are limited as the virus is particularly resistant to common disinfectants (4). At present, two commercially available vaccines, Rotarix (GlaxoSmithKline plc) and RotaTeq (Merck $\mathrm{KGaA}$ ), have been approved in many countries (5). These vaccines seem to be effective at reducing rotavirus-induced diarrhea. However, the vaccines only exert positive effects against the target strain of the virus, and the immunization schedule of vaccines must be strictly controlled (6). Therefore, it is important to develop other natural antiviral agents to treat rotavirus infection.

Macleaya cordata extract is a member of the Macleaya genus in the family Papaveraceae, which contains various active alkaloids (7). M. cordata has been reported to have a wide spectrum of biological activities, including antiviral, anti-inflammatory, antioxidative, detoxifying and antimicrobial effects (8). In China, $M$. cordata has also been used as a traditional medicinal herb to treat cancer, including thyroid cancer and cervical cancer (9). M. cordata is also a traditional medicinal plant in Europe and North America, used for treating ringworm infection and insect bites (8).

The present study hypothesized that $M$. cordata may play key roles in mediating antiviral responses and consequently reduce the incidence of rotavirus-induced diarrhea. The aim of the present study was to assess the biological influence of $M$. cordata on rotavirus-induced diarrhea by detecting rotavirus antigens and inflammatory cytokines, evaluating the histopathological changes in the small intestine and exploring the underlying mechanisms by which $M$. cordata mediates its antiviral and anti-inflammatory effects. 


\section{Materials and methods}

Cells and virus. Simian rotavirus SA11 and rhesus monkey kidney MA-104 cells (American Type Culture Collection) were used for rotavirus infection and cultivation (10). MA-104 cells were grown in DMEM (Beyotime Institute of Biotechnology) supplemented with 10\% FBS (Beyotime Institute of Biotechnology) at $37^{\circ} \mathrm{C}$ under $5 \% \mathrm{CO}_{2}$. SA11 was activated with $15 \mu \mathrm{g} / \mathrm{ml}$ trypsin for $30 \mathrm{~min}$ and cultured in MA-104 cells for $48 \mathrm{~h}$. The virus titer used in the present study was $4 \times 10^{7}$ focus-forming units $/ \mathrm{ml}$ (11).

Animals and experimental design. A total of 72 newborn BALB/c mice (female, 16-18 g) were obtained from Nanjing Medical University Animal Laboratory and maintained as previously described (12). Animal procedures were in line with the National Institutes of Health Guidelines for the Care and Use of Laboratory Animals and were approved by the Animal Committee of the Jiangsu Agri-animal Husbandry Vocational College. All mice were negative for rotavirus antibodies before rotavirus exposure. Mice were randomly assigned to 6 groups ( $\mathrm{n}=12$ in each group) and ear-coded as follows: Control; rotavirus (virus-inoculated and administered saline); virus-inoculated and administered $1 \mathrm{mg} / \mathrm{kg} / \mathrm{day} M$. cordata (Phytobiotics Futterzusatzstoffe $\mathrm{GmbH}$ ); virus-inoculated and administered $2 \mathrm{mg} / \mathrm{kg} / \mathrm{day} M$. cordata; virus-inoculated and administered with $4 \mathrm{mg} / \mathrm{kg} / \mathrm{day} M$. cordata; and virus-inoculated and administered with $4 \mathrm{mg} / \mathrm{kg} / \mathrm{d}$ ribavirin (Sigma-Aldrich; Merck $\mathrm{KGaA}$ ). At 3 days of age, mice were inoculated with $30 \mu \mathrm{l}$ rotavirus-infected MA-104 cells via oral gavage. At 2 days post-inoculation (DPI 2), the mice showed signs of diarrhea. Inoculated mice were evaluated for diarrhea ( 0 to 4$)$ determined as the following: No fecal discharge recorded, score 0 ; brown molded stool, score 1; brown soft stool, score 2; soft yellow stool, score 3; yellow watery stool, score 4; and perianal stool contamination, also score 4 . 1 was considered no diarrhea; 2 was considered common diarrhea; 3 was considered severe diarrhea; and 4 was considered very severe diarrhea. Mice with a score $>2$ were identified as having diarrhea. The mice were treated with $M$. cordata $(1,2,4 \mathrm{mg} / \mathrm{kg} / \mathrm{day})$ or ribavirin $(4 \mathrm{mg} / \mathrm{kg} /$ day). The antiviral effects of $M$. cordata were evaluated on fecal material, serum and small intestine histology. At the predetermined times, mice were anesthetized prior to euthanasia by decapitation. The experiments were based on humane endpoint criteria, that mice with a score $>2$ were identified. All the animal procedures were in accordance with the National Institutes of Health Guidelines for the Care and Use of Laboratory Animals. The small intestines were harvested at DPI 3 for pathological analysis $(n=6)$. Samples were quickly obtained and frozen in liquid nitrogen and stored at $-80^{\circ} \mathrm{C}$ for further analysis. The remaining six mice in each group were sacrificed at DPI 7 for diarrhea observation and the detection of inflammatory cytokines.

Diarrhea score. To evaluate the antiviral effects of M.cordata, fecal consistency scores (1, normal; 2 , soft feces; 3 , mild diarrhea; 4 , severe diarrhea) were determined for each rat (13). Scores $\geq 2$ were considered a symptom of diarrhea. The severity of diarrhea was calculated by dividing the total number of all scores by the number of total experimental mice.
ELISA. Fecal samples were collected at DPI 3, dissolved in PBS and centrifuged at $1,000 \mathrm{xg}$ for $15 \mathrm{~min}$ at $4^{\circ} \mathrm{C}$. The supernatant was collected for rotavirus antigen detection using an ELISA kit (cat. no. CSB-EQ027718MO; Cusabio Technology, LLC) according to the manufacturer's protocol. The absorbance was read at a wavelength of $450 \mathrm{~nm}$ using an EL808 microplate reader (BioTek Instruments, Inc.). An optical density at $450 \mathrm{~nm}$ of the sample minus that of the control group $\geq 0.1$ was considered to be positive.

Macrophage migration inhibitory factor (MIF) (cat. no. abx258931; Biolead Co., Ltd.), interleukin (IL)-8 (cat. no. KHC0081; Invitrogen; Thermo Fisher Scientific, Inc.), IL-10 (cat. no. 29-8101-65; eBioscience; Thermo Fisher Scientific, Inc.), IL- $\beta$ (cat. no. BMS224-2; Invitrogen; Thermo Fisher Scientific, Inc.), interferon (IFN) $\gamma$ (cat. no. E-EL-R0009c; Elabscience), tumor necrosis factor (TNF)- $\alpha$ (cat. no. BMS223HS; Invitrogen; Thermo Fisher Scientific, Inc.) and IL-6 (cat. no. BMS213HS; Invitrogen; Thermo Fisher Scientific, Inc.) in serum were also determined using commercial ELISA kits. A total of $500 \mu \mathrm{l}$ of blood was collected each day and centrifuged at $1,000 \mathrm{x}$ g for $10 \mathrm{~min}$ at $4^{\circ} \mathrm{C}$ to obtain serum samples.

Reverse transcription-quantitative $P C R$ (RT-qPCR). Rotavirus VP6 RNA in fecal samples was extracted as previously described (14). Total RNA was extracted using a QIAamp Viral RNA Mini kit (cat. no. 52904; Qiagen China Co., Ltd.) and stored at $-80^{\circ} \mathrm{C}$ for further analysis. RT was performed under the following thermocycling conditions: $61^{\circ} \mathrm{C}$ for $3 \mathrm{~min}$, followed by $95^{\circ} \mathrm{C}$ for $5 \mathrm{~min}$ and chilling at $4^{\circ} \mathrm{C}$. The gene expression levels were measured using the SYBR Premix Ex TaqTM (Takara Bio, Inc.). The temperature protocol was as follow: $95^{\circ} \mathrm{C}$ for $5 \mathrm{sec}, 60^{\circ} \mathrm{C}$ for $45 \mathrm{sec}$ and $72^{\circ} \mathrm{C}$ for $1 \mathrm{~min}$ for 40 cycles using a Real Time PCR system (Illumina, Inc.). A standard curve established using Light-Cycler 480 gene scanning software V1.5 (Roche) was used to measure RNA concentration by performing linear analysis of the data. The following primer pair was used for the qPCR: The amount of rotavirus RNA in fecal samples was quantified based on the standard curve derived from 10 -fold serial dilutions of plasmid DNA, which was used as an external standard for all RT-qPCR experiments (11). The data were calculated using the $2^{-\Delta \Delta C T}$ method (12).

Hematoxylin and eosin $(H \& E)$ staining. Mouse small intestines were excised at DPI 3, perfused with $10 \%$ formalin for $24 \mathrm{~h}$ at $4^{\circ} \mathrm{C}$ and embedded in paraffin. Sections with a thickness of $5 \mu \mathrm{m}$ were stained with $0.5 \%$ hematoxylin for $5 \mathrm{~min}$ at room temperature, followed by staining with $0.5 \%$ eosin solution for $1 \mathrm{~min}$ at room temperature. The images were examined under a light microscope (Olympus Corporation) at x200 magnification.

TUNEL assay. To evaluate the effects of M. cordata on intestinal epithelial cell apoptosis, mice were sacrificed at DPI 3. Small intestine samples were fixed in $10 \%$ formalin for $24 \mathrm{~h}$ at $4^{\circ} \mathrm{C}$ and embedded in paraffin. Sections with $5-\mu \mathrm{m}$ thickness were used for TUNEL staining (according to the instructions of In Situ Cell Death Detection kit (cat. no. 11684817910; Roche Diagnostics $\mathrm{GmbH}$ ). In brief, the sections were washed 
and incubated with proteinase $\mathrm{K}$ for $30 \mathrm{~min}$ at $37^{\circ} \mathrm{C}$, followed by incubation with a terminal deoxynucleotidyl transferase. Then the sections were treated with $3 \%$ hydrogen peroxide for 5 min and subsequently incubated with the peroxidase-conjugated antibodies from the aforementioned kit, for $10 \mathrm{~min}$ at room temperature. Then the DAB solution, with $3 \%$ hydrogen peroxide and the methyl green was added for $2 \mathrm{~min}$ at room temperature. After treating with Mayer's hematoxylin for $1 \mathrm{~min}$ at room temperature, the TUNEL-positive cells were observed under a light microscope (Olympus Corporation) at x200 magnification. Five fields of view were selected randomly from one section.

Western blotting. Total protein was extracted from small intestines using RIPA buffer (Beyotime Institute of Biotechnology) and the concentration was detected using the BCA kit (Beyotime Institute of Biotechnology). Proteins (30 $\mu \mathrm{g}$ per lane) were separated by $10 \%$ SDS-PAGE and transferred to a PVDF membrane (EMD Millipore). Subsequently, the membrane was blocked with 5\% non-fat milk for $2 \mathrm{~h}$ at room temperature. The membrane was incubated overnight at $4^{\circ} \mathrm{C}$ with the following primary antibodies: Janus kinase 2 (JAK2; 1:1,000; cat. no. 74987), phosphorylated (p)-JAK2 (1:750; cat. no. 66245), STAT3 (1:1,000; cat. no. 9139), p-STAT3 (1:750; cat. no. 4113) and GAPDH (1:2,000; cat. no. 97116). The membranes were subjected to three 5-min washes with $0.5 \%$ TBS with Tween-20, then probed with HRP-conjugated secondary mouse antibodies (1:2,000; cat. no. 7076) for $1 \mathrm{~h}$ at room temperature. Following washing, protein levels were examined with an ECL kit (Beyotime Institute of Biotechnology). All antibodies were purchased from CST Biological Reagents Co., Ltd. The gray values of the bands were calculated using ImageJ software (version 4.3; National Institutes of Health) with GAPDH as the loading control.

Immunohistochemical analysis. At DPI 3, the aforementioned paraffin-embedded sections were used to assess the expression of p-JAK2 and p-STAT3 based immunohistochemical staining. Sections with $5-\mu \mathrm{m}$ thickness were incubated with primary antibodies for JAK2 (1:1,000; cat. no. 74987), p-JAK2 (1:750; cat. no. 66245), STAT3 (1:1,000; cat. no. 9139) and p-STAT3 (1:750; cat. no. 4113$)$ at $37^{\circ} \mathrm{C}$ overnight, followed by incubation with biotinylated secondary antibody (cat. no. 31926 , $30-40 \mu \mathrm{l}$ ) at room temperature for $30 \mathrm{~min}$. All antibodies were purchased from CST Biological Reagents Co., Ltd. All images were captured under a light microscope (Olympus Corporation) at $\mathrm{x} 200$ magnification.

Statistical analysis. Data were analyzed using GraphPad Prism 5.0 (GraphPad Software, Inc.) and are expressed as the mean \pm SD. All experiments were repeated at least three times. One-way ANOVA followed by Tukey's post hoc test was used to compare the differences between groups. $\mathrm{P}<0.05$ was considered to indicate a statistically significant difference.

\section{Results}

M. cordata decreases the severity of diarrhea in rotavirusinfected newborn mice. To investigate the effects of $M$. cordata on rotavirus infection in vivo, newborn mice were inoculated with SA11 rotavirus cultured in MA104 cells by oral gavage. As expected, mice without rotavirus treatment did not exhibit diarrhea during the entire experimental period, whereas mice inoculated with rotavirus presented with severe diarrhea at DPI 2 (Fig. 1A). Diarrhea was inhibited after ribavirin treatment ranging from DPI 4-7, when compared to Rotavirus-treated group, as evidenced by the decreased diarrhea score in the ribavirin group. $M$. cordata treatment resulted in decreased diarrhea scores at DPI 4 compared with rotavirus-inoculated mice, and diarrhea scores appeared reduced at DPI 6. No significant difference was observed in the diarrhea scores between $M$. cordata- and ribavirin-treated mice. The results demonstrated that $M$. cordata could effectively inhibit rotavirus-induced diarrhea.

M. cordata inhibits rotavirus replication in rotavirus-infected newborn mice. In order to determine whether M. cordata suppressed rotavirus infectivity, rotavirus VP6 RNA and antigen expression were evaluated using RT-qPCR and ELISA, respectively. No expression of rotavirus antigens was detected in mouse feces prior to the experiments. As depicted in Fig. 1B, no increase was measured in the rotavirus copy number of control mice, whereas $M$. cordata remarkedly reduced rotavirus copy numbers at the three tested doses. Similarly, the $M$. cordata- and ribavirin-treated groups presented with reduced rotavirus antigen levels compared with virus-inoculated mice (Fig. 1C). No significant difference in antigen levels between the $M$. cordata ( $4 \mathrm{mg} / \mathrm{kg} /$ day) and ribavirin-treated groups was measured.

M. cordata restores the levels of inflammatory cytokines in rotavirus-infected newborn mice. ELISA was performed to quantify inflammatory cytokine levels in rotavirus-infected newborn mice. As shown in Fig. 2, the levels of the pro-inflammatory cytokines MIF, IL- 6 , IL- 8 , TNF- $\alpha$, IFN- $\beta$ and IFN- $\gamma$ increased, but the levels of anti-inflammatory IL-10 declined, after rotavirus infection. However, M. cordata or ribavirin treatment significantly reversed the upregulation of pro-inflammatory mediator levels and the downregulation of anti-inflammatory molecule levels $(\mathrm{P}<0.05$; Fig. 2$)$. The results indicated that $M$. cordata may exert immunomodulatory and anti-inflammatory roles in rotavirus-induced diarrhea.

M. cordata induces histopathological changes in the small intestine. H\&E staining was used to examine the effects of $M$. cordata on rotavirus-induced changes in the pathology of the small intestine. As shown in Fig. 3A, enterocytes were clearly polarized and the nuclei were localized at the base of the enterocytes in control mice. Virus-inoculated mice showed notable lesions in the small intestinal villi, including epithelium defluxion, edema and vacuolar degeneration. However, M. cordata and ribavirin treatment led to an attenuation of the lesions in the small intestinal villi. These data indicated that $M$. cordata treatment could improve the pathological damage in the small intestine caused by rotavirus.

M. cordata suppresses enterocyte apoptosis in rotavirus-infected newborn mice. A TUNEL assay was performed to further investigate the potential effect of $M$. cordata on enterocyte apoptosis in rotavirus-induced mice. 

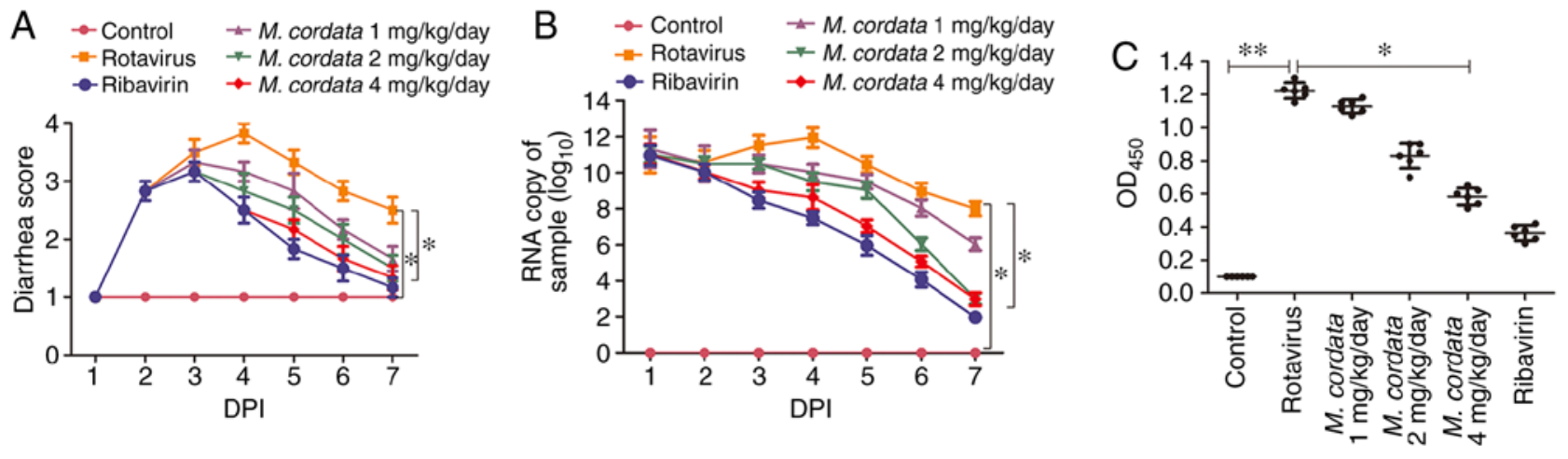

Figure 1. Diarrhea scores and viral antigen shedding experiments. (A) Diarrhea scores of mice treated with different concentrations of $M$. cordata following rotavirus induction. (B) Quantification of viral RNA copy numbers in the fecal samples of mice. (C) Virus antigen levels in the fecal samples of mice. " $\mathrm{P}<0.05$; ${ }^{* *} \mathrm{P}<0.01$. DPI, days post-inoculation; OD, optical density; M. cordata, Macleaya cordata.
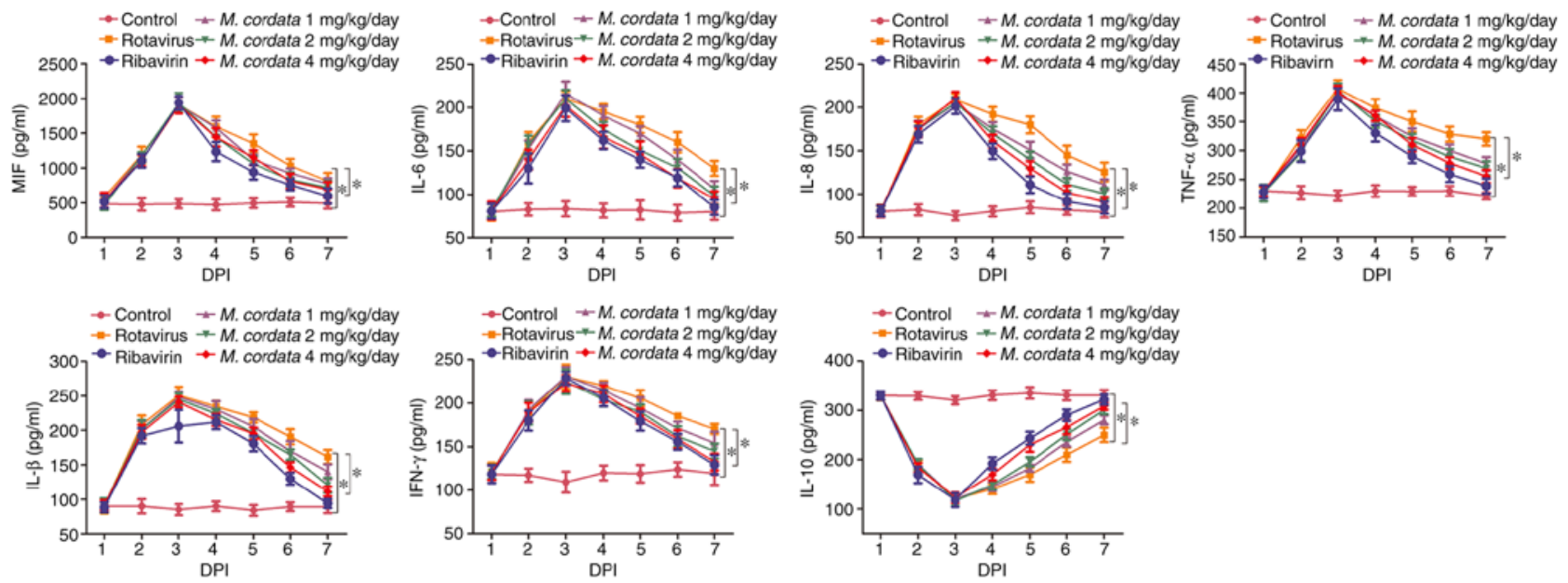

Figure 2. Influence of $M$. cordata on rotavirus-induced levels of IL-8, IFN- $\beta$, IFN- $\gamma$, TNF- $\alpha$, IL-6 and IL-10 in mice. "P<0.05. MIF, migration inhibitory factor; IL, interleukin; IFN, interferon; TNF, tumor necrosis factor; DPI, days post-inoculation; M. cordata, Macleaya cordata.

Virus-inoculated mice showed notable enterocyte apoptosis compared with control mice (Fig. 3B). However, M. cordata treatment resulted in reduced apoptotic cell death as confirmed by the decreased number of TUNEL-positive cells, indicating that enterocyte apoptosis was repressed in rotavirus-infected mice.

M. cordata exerts antiviral effects via the JAK2/STAT3 pathway in vivo. To verify whether $M$. cordata regulated the activity of the JAK2/STAT3 signaling pathway, the protein expression levels of JAK 2 and STAT3 were detected by western blotting and immunohistochemical assays. As shown in Fig. 4A, rotavirus increased the levels of p-JAK2 and p-STAT3 compared with the control, whereas the levels of p-JAK2 and p-STAT3 markedly decreased following $M$. cordata treatment compared with rotavirus-induced samples $(\mathrm{P}<0.05)$. M.cordata was found to markedly decrease the Rotavirus-induced phosphorylation of JAK2 and STAT3. On the other hand, no significant differences existed between the $M$. cordata and ribavirin-treated groups, indicating a comparable therapeutic effect of M. cordata to ribavirin. Consistent with the western blotting results, immunohistochemical analysis also revealed that $M$. cordata markedly reversed the increased expression levels of p-JAK2 and p-STAT3 induced by rotavirus (Fig. 4B). Overall, the results suggested that $M$. cordata may regulate antiviral and inflammatory responses by inactivating the JAK2/STAT3 signaling pathway.

\section{Discussion}

Rotavirus infection can result in severe diarrhea with high morbidity and mortality (15). However, no specific drug against rotavirus infection is currently available. Therefore, herbal products can be potentially used as therapeutic drugs against rotavirus-induced diarrhea. In the present study, M. cordata, a traditional medicinal herb, showed an antiviral role in mice with rotavirus-induced diarrhea.

The employment of rotavirus-infected animal models has been used to better understand the pathogenesis of rotavirus-associated diseases $(16,17)$. The physiopathology of rotavirus-induced diarrhea has been investigated in different animal species including cows, pigs, sheep, rabbits and mice (18). Murine models, which are cost-effective, easy to maintain and fast-breeding, have been frequently used to study rotavirus infection (19). In the present study, a rotavirus-infected mouse model was established by inoculating SA11 virus 


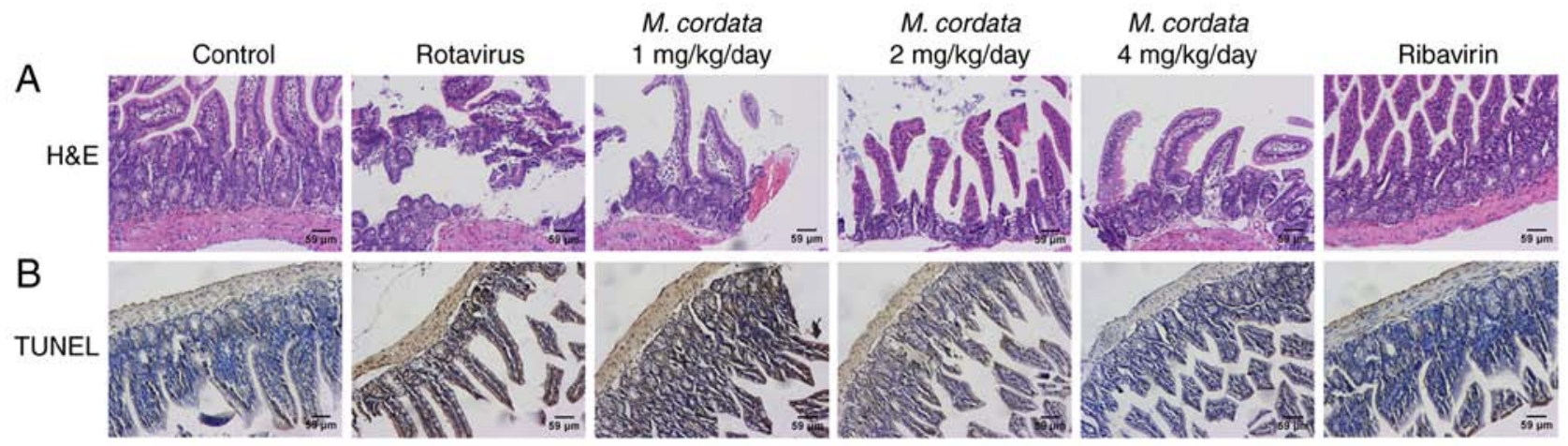

Figure 3. M. cordata induces histopathological changes in the small intestine. (A) H\&E evaluation of small intestine samples of mice (magnification, $\mathrm{x} 200$ ). (B) TUNEL assay showing the effects of $M$. cordata on intestinal epithelial cell apoptosis of mice (magnification, x200). H\&E, hematoxylin and eosin; M. cordata, Macleaya cordata.
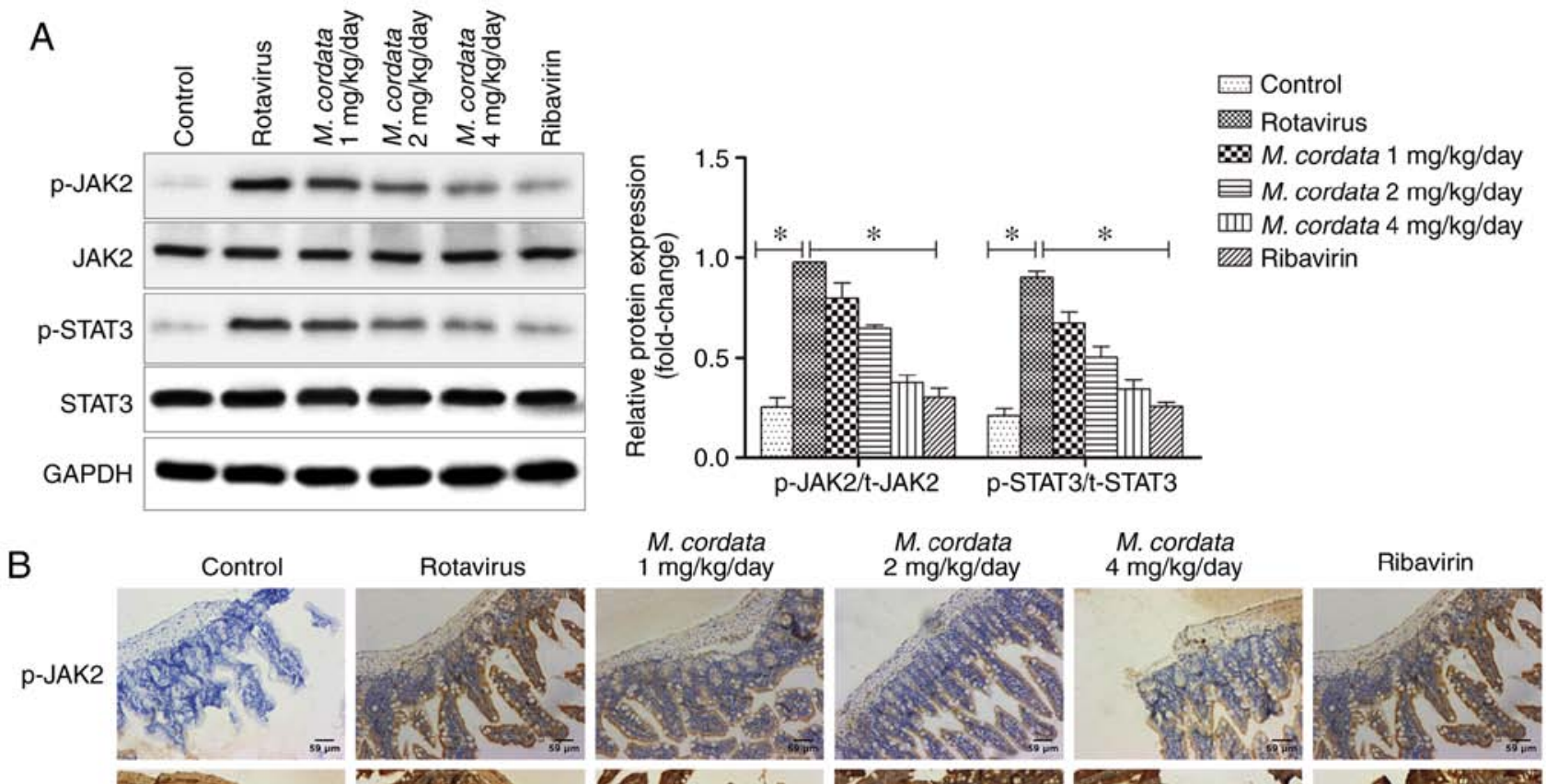

JAK2
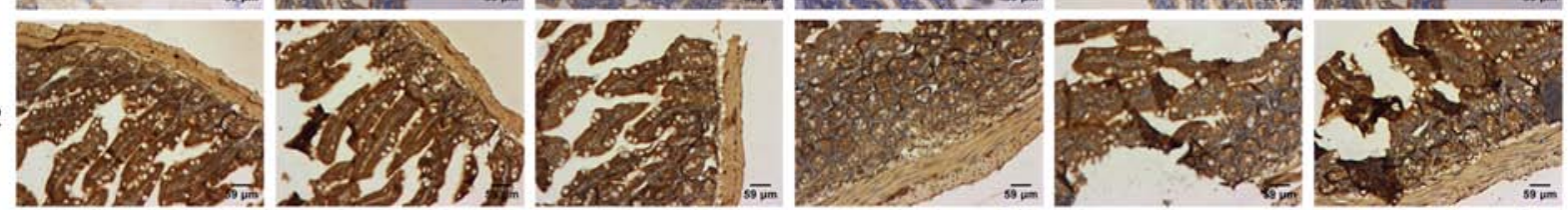

p-STAT3
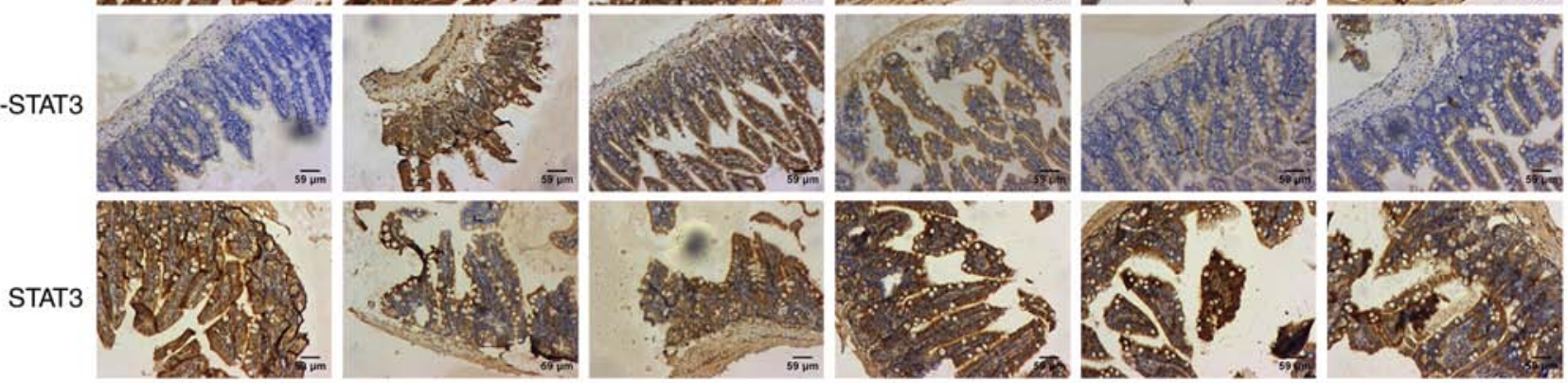

Figure 4. Effects of M. cordata on the JAK2/STAT3 pathway. (A) Protein levels of JAK2, p-JAK2, STAT3 and p-STAT3 detected by western blotting. (B) Levels of JAK2, p-JAK2, STAT3 and p-STAT3 detected by immunohistochemical analysis (magnification, x200). "P<0.05. JAK2, Janus kinase 2; p, phosphorylated; t, total; . cordata, Macleaya cordata.

passaged in MA104 cells into newborn mice. A previous study showed that targets of 26 isoquinoline alkaloids were identified from $M$. cordata therefore the exact active ingredient(s) in this present study remain unclear (20). The targets in the interaction network of $M$. cordata were implicated in various biological progresses including cancer development, neurodegeneration, 
inflammation and autoimmunity, parasitosis, injury and pain (20,21). Among these targets, dihydrochelerythrine (C6) was found to hit 23 targets, while MIF was found to hit 15 alkaloids (C1-2, C11-16, C19-25), and appeared to be the most promising target related to cancer (20). These findings further demonstrated that M. cordata had pleiotropic antiviral, anticancer and immunomodulatory effects. In the present study, M. cordata suppressed the diarrhea caused by rotavirus infection. In addition, $M$. cordata inhibited virus replication, since it reduced the fecal shedding of rotavirus RNA and antigens in mice. The present findings corroborate the results of a previous study, in which $M$. cordata was found to alleviate rotavirus-induced diarrhea (14).

Rotavirus infection is known to affect the secretion of inflammatory cytokines (22). M. cordata was demonstrated to have immunomodulatory properties and anti-inflammatory effects (23). M. cordata was shown to stimulate the anti-inflammatory enzyme heme oxygenase, which is associated with the pathogenesis of autoimmune diseases $(24,25)$. The inflammatory response is characterized by the release of inflammatory cytokines $(26,27)$. MIF, a widely expressed pleiotropic proinflammatory cytokine, is a part of the innate immune response to inflammatory diseases $(28,29)$. MIF promotes the release of inflammatory indicators such as IL-6, IL-1 and TNF- $\alpha$ (30). In the present study, rotavirus infection elevated MIF levels compared with the control group, whereas $M$. cordata treatment significantly reduced MIF levels compared with rotavirus-induced samples. Subsequently, the levels of inflammatory cytokines including IL- 8 , IFN- $\beta$, IFN- $\gamma$, TNF- $\alpha$ and IL-10 were determined. Similar to MIF, rotavirus infection led to increased levels of the pro-inflammatory cytokines IL-8, IFN- $\beta$, IFN- $\gamma$ and TNF- $\alpha$, as well as decreased levels of the anti-inflammatory cytokine IL-10, whereas $M$. cordata reversed the levels of inflammatory cytokines in the serum of mice with rotavirus-induced diarrhea, which is in accordance with previous reports $(23,2)$.

Considering that rotavirus was documented to affect histopathological changes of the small intestine $(31,32)$, the pathology of the small intestine was determined by histological analysis. Rotavirus-induced lesions in the small intestine were markedly restored following $M$. cordata or ribavirin treatment. The TUNEL assay results further confirmed that rotavirus promoted epithelial cell apoptosis, and M. cordata or ribavirin treatment reversed the apoptosis induced by rotavirus infection.

Traditional Chinese medicine may improve inflammatory processes by regulating various protein kinases, such as mitogen-activated protein kinase, PI3K and AKT (33). $M$. cordata exerts an important role in the development of immunoinflammatory diseases and cancer by inhibiting the PI3K/AKT/mTOR pathway $(34,35)$. The PI3K/AKT/mTOR axis is involved in the pathogenesis of rotavirus infection (36). The JAK2/STAT3 pathway is an important intracellular signal transduction pathway; phosphorylation of JAK2 results in STAT3 phosphorylation, which consequently regulates the transcription of target genes encoding inflammatory cytokines (37). Previous studies confirmed that the JAK2/STAT3 signaling pathway is not only associated with various important functions in normal and malignant cells including differentiation, proliferation, angiogenesis and apoptosis (38), but is also confirmed as a pivotal inflammatory and immune mediator during disease progression, including hyperglycemia-associated inflammation, atherosclerosis and rheumatoid arthritis (39-41). Aberrant activation of the JAK2/STAT3 pathway generally occurs during the process of neuroinflammatory disease (42). In the present study, mice exhibited a significant activation in JAK2/STAT3 phosphorylation following rotavirus infection compared with the control group, while $M$. cordata treatment reduced the levels of p-JAK2 and p-STAT3. The immunohistochemistry results also demonstrated reduced expression of p-JAK2 and p-STAT3 following $M$. cordata treatment. These data suggested that the JAK2/STAT3 signaling pathway was inhibited following $M$. cordata or ribavirin treatment. These findings are in accordance with previous research, which reported that the JAK2/STAT3 pathway was involved in the inflammatory response by inducing chemokine release (43-45).

In conclusion, $M$. cordata was demonstrated to exert antiviral and anti-inflammatory effects, which protected against rotavirus-induced diarrhea. Furthermore, the present findings showed that $M$. cordata repressed rotavirus-induced diarrhea by suppressing the activation of the JAK2/STAT3 signaling pathway. These results suggested that $M$. cordata could be used as a therapeutic agent for rotavirus-induced diarrhea.

\section{Acknowledgements}

Not applicable.

\section{Funding}

This work was supported by the Jiangsu Province Agricultural Three New Projects [grant no. SXGC (2017)249], Taizhou Agricultural Technology Supporting Project (grant no. TN201711) and Jiangsu Agri-animal Husbandry Vocational College project (grant no. NSF201901).

\section{Availability of data and materials}

The datasets used and/or analyzed during the present study are available from the corresponding author on reasonable request.

\section{Authors' contributions}

$\mathrm{CJ}$ and CW conceived and planned the experiments of this study. CJ performed the experiments. HY and XC performed and analyzed the ELISA and reverse transcription-quantitative PCR experiments. SQ, BZ and LJ performed the hematoxylin and eosin, TUNEL, immunohistochemistry and western blot analyses. CW supervised the project. CJ and HY wrote the manuscript. All authors analyzed and interpreted the data. All authors contributed to and approved the final version of the manuscript.

\section{Ethics approval and consent to participate}

Animal procedures were in line with the National Institutes of Health Guidelines for the Care and Use of Laboratory Animals and were approved by the Animal Committee of the Jiangsu Agri-animal Husbandry Vocational College. 


\section{Patient consent for publication}

Not applicable.

\section{Competing interests}

The authors declare that they have no competing interests.

\section{References}

1. Boshuizen JA, Reimerink JH, Korteland-van Male AM, van Ham VJ, Koopmans MP, Büller HA, Dekker J and Einerhand AW: Changes in small intestinal homeostasis, morphology, and gene expression during rotavirus infection of infant mice. J Virol 77: 13005-13016, 2003.

2. Tate JE, Burton AH, Boschi-Pinto C, Steele AD, Duque J and Parashar UD; WHO-coordinated Global Rotavirus Surveillance Network: 2008 estimate of worldwide rotavirus-associated mortality in children younger than 5 years before the introduction of universal rotavirus vaccination programs: A systematic review and meta-analysis. Lancet Infect Dis 12: 136-141, 2012.

3. Pesavento JB, Crawford SE, Estes MK and Prasad BV: Rotavirus proteins: Structure and assembly. Curr Top Microbiol Immunol 309: 189-219, 2006.

4. Kawahara T, Tomono T, Hamauzu Y, Tanaka K and Yasui H: Inhibitory effect of a hot-water extract of leaves of Japanese big-leaf magnolia (magnolia obovata) on rotavirus-induced diarrhea in mouse pups. Evid Based Complement Alternat Med 2014: 365831, 2014.

5. Anderson EJ: Rotavirus vaccines: Viral shedding and risk of transmission. Lancet Infect Dis 8: 642-649, 2008.

6. Lepage P and Vergison A: Prevention of childhood rotavirus disease through the use of Rotarix and RotaTeq vaccines. Expert Opin Biol Ther 7: 1881-1892, 2007.

7. Liu M, Lin YL, Chen XR, Liao CC and Poo WK: In vitro assessment of Macleaya cordata crude extract bioactivity and anticancer properties in normal and cancerous human lung cells. Exp Toxicol Pathol 65: 775-787, 2013.

8. Lin L, Liu YC, Huang JL, Liu XB, Qing ZX, Zeng JG and Liu ZY: Medicinal plants of the genus Macleaya (Macleaya cordata, Macleaya microcarpa): A review of their phytochemistry, pharmacology, and toxicology. Phytother Res 32: 19-48, 2018

9. Ke W, Lin X, Yu Z, Sun Q and Zhang Q: Molluscicidal activity and physiological toxicity of Macleaya cordata alkaloids components on snail oncomelania hupensis. Pestic Biochem Physiol 143 111-115, 2017.

10. Arnold M, Patton JT and McDonald SM: Culturing, storage, and quantification of rotaviruses. Curr Protoc Microbiol 15: Unit 15C 3, 2009.

11. Tian Z, Shen Z, He H, Zhang J, Li J and Wu Y: Antiviral effects of cyclosporin a in neonatal mice with rotavirus-induced diarrhea. J Pediatr Gastroenterol Nutr 60: 11-17, 2015.

12. Livak KJ and Schmittgen TD: Analysis of relative gene expression data using real-time quantitative PCR and the 2(-Delta Delta C(T)) method. Methods 25: 402-408, 2001.

13. Shen $\mathrm{Z}, \mathrm{He} \mathrm{H}, \mathrm{Wu} \mathrm{Y}$ and $\mathrm{Li} \mathrm{J}$ : Cyclosporin a inhibits rotavirus replication and restores interferon-beta signaling pathway in vitro and in vivo. PLoS One 8: e71815, 2013.

14. Liu G, Guan G, Fang J, Martínez Y, Chen S, Bin P, Duraipandiyan V, Gong T, Tossou MC, Al-Dhabi NA and Yin Y: Macleaya cordata extract decreased diarrhea score and enhanced intestinal barrier function in growing piglets. Biomed Res Int 2016: 1069585, 2016.

15. Dung TT, Phat VV, Nga TV, My PV, Duy PT, Campbell JI, Thuy CT, Hoang NV, Van Minh P, Le Phuc H, et al: The validation and utility of a quantitative one-step multiplex RT real-time PCR targeting rotavirus A and norovirus. J Virol Methods 187: $138-143,2013$

16. Alfajaro MM, Kim HJ, Park JG, Ryu EH, Kim JY, Jeong YJ, Kim DS, Hosmillo M, Son KY, Lee JH, et al: Anti-rotaviral effects of Glycyrrhiza uralensis extract in piglets with rotavirus diarrhea. Virol J 9: 310, 2012.

17. Ciarlet M, Conner ME, Finegold MJ and Estes MK: Group A rotavirus infection and age-dependent diarrheal disease in rats: A new animal model to study the pathophysiology of rotavirus infection. J Virol 76: 41-57, 2002.
18. Guerin-Danan C, Meslin JC, Lambre F, Charpilienne A, Serezat M, Bouley C, Cohen J and Andrieux C: Development of a heterologous model in germfree suckling rats for studies of rotavirus diarrhea. J Virol 72: 9298-9302, 1998.

19. Ward RL: Possible mechanisms of protection elicited by candidate rotavirus vaccines as determined with the adult mouse model. Viral Immunol 16: 17-24, 2003.

20. Lei Q, Liu H, Peng Y and Xiao P: In silico target fishing and pharmacological profiling for the isoquinoline alkaloids of Macleaya cordata (Bo Luo Hui). Chin Med 10: 37, 2015.

21. Freitas K, Carroll FI and Damaj MI: The antinociceptive effects of nicotinic receptors $\alpha 7$-positive allosteric modulators in murine acute and tonic pain models. J Pharmacol Exp Ther 344: 264-275, 2013.

22. Jiang B, Snipes-Magaldi L, Dennehy P, Keyserling H, Holman RC, Bresee J, Gentsch J and Glass RI: Cytokines as mediators for or effectors against rotavirus disease in children. Clin Diagn Lab Immunol 10: 995-1001, 2003.

23. Khadem A, Soler L, Everaert N and Niewold TA: Growth promotion in broilers by both oxytetracycline and Macleaya cordata extract is based on their anti-inflammatory properties. Br J Nutr 112: 1110-1118, 2014.

24. Vrba J, Orolinova E and Ulrichova J: Induction of heme oxygenase- 1 by Macleaya cordata extract and its constituent sanguinarine in RAW264.7 cells. Fitoterapia 83: 329-335, 2012.

25. Fagone P, Patti F, Mangano K, Mammana S, Coco M, Touil-Boukoffa C, Chikovani T, Di Marco R and Nicoletti F: Heme oxygenase-1 expression in peripheral blood mononuclear cells correlates with disease activity in multiple sclerosis J Neuroimmunol 261: 82-86, 2013.

26. Ronco C, Ronco F and Mccullough PA: A call to action to develop integrated curricula in cardiorenal medicine. Rev Cardiovasc Med 18: 93-99, 2017.

27. Jin J, Liu Y, Huang L and Tan H: Advances in epigenetic regulation of vascular aging. Rev Cardiovasc Med 20: 19-25, 2019.

28. Yilmaz E, Coskun EI, Gul M, Sahin N, Tuncay G and Simsek Y: Nuclear factor-kappa $\beta$ pathway and endometrial cancer: A pilot study. Eur J Gynaecol Oncol 38: 536-540, 2017.

29. Tian JS, Zhai QJ, Zhao Y, Chen R and Zhao LD: 2-(2-benzofuranyl)-2-imidazoline (2-BFI) improved the impairments in AD rat models by inhibiting oxidative stress, inflammation and apoptosis. J Integr Neurosci 16: 385-400, 2017.

30. Fagone P, Mazzon E, Cavalli E, Bramanti A, Petralia MC, Mangano K, Al-Abed Y, Bramati P and Nicoletti F: Contribution of the macrophage migration inhibitory factor superfamily of cytokines in the pathogenesis of preclinical and human multiple sclerosis: In silico and in vivo evidences. J Neuroimmunol 322: 46-56, 2018.

31. Presti M, Mazzon E, Basile MS, Petralia MC, Bramanti A, Colletti G, Bramanti P, Nicoletti F and Fagone P: Overexpression of macrophage migration inhibitory factor and functionally-related genes, D-DT, CD74, CD44, CXCR2 and CXCR4, in glioblastoma. Oncol Lett 16: 2881-2886, 2018.

32. Lang T, Lee JPW, Elgass K, Pinar AA, Tate MD, Aitken EH, Fan H, Creed SJ, Deen NS, Traore DAK, et al: Macrophage migration inhibitory factor is required for NLRP3 inflammasome activation. Nat Commun 9: 2223, 2018.

33. Gandhi GR, Santos VS, Denadai M, da Silva Calisto VK, de Souza Siqueira Quintans J, de Oliveira E Silva AM, de Souza Araújo AA, Narain N, Cuevas LE, Júnior LJQ and Gurgel RQ: Cytokines in the management of rotavirus infection: A systematic review of in vivo studies. Cytokine 96: 152-160, 2017.

34. Feng N, Kim B, Fenaux M, Nguyen H, Vo P, Omary MB and Greenberg HB: Role of interferon in homologous and heterologous rotavirus infection in the intestines and extraintestinal organs of suckling mice. J Virol 82: 7578-7590, 2008.

35. Liu M, Zhao G, Cao S, Zhang Y, Li X and Lin X: Development of certain protein kinase inhibitors with the components from traditional chinese medicine. Front Pharmacol 7: 523, 2017.

36. Mammana S, Bramanti P, Mazzon E, Cavalli E, Basile MS, Fagone P, Petralia MC, McCubrey JA, Nicoletti F and Mangano K: Preclinical evaluation of the PI3K/Akt/mTOR pathway in animal models of multiple sclerosis. Oncotarget 9: 8263-8277, 2018

37. Horie R, Nakamura O, Yamagami Y, Mori M, Nishimura H, Fukuoka $\mathrm{N}$ and Yamamoto T: Apoptosis and antitumor effects induced by the combination of an mTOR inhibitor and an autophagy inhibitor in human osteosarcoma MG63 cells. Int J Oncol 48: 37-44, 2016. 
38. Yin Y, Dang W, Zhou X, Xu L, Wang W, Cao W, Chen S, Su J, Cai X, Xiao S, et al: PI3K-Akt-mTOR axis sustains rotavirus infection via the 4E-BP1 mediated autophagy pathway and represents an antiviral target. Virulence 9: 83-98, 2018.

39. Raible DJ, Frey LC and Brooks-Kayal AR: Effects of JAK2-STAT3 signaling after cerebral insults. JAKSTAT 3: e29510, 2014.

40. Sansone $P$ and Bromberg J: Targeting the interleukin6/Jak/stat pathway in human malignancies. J Clin Oncol 30: 1005-1014, 2012.

41. Cui J, Zhang F, Cao W, Wang Y, Liu J, Liu X, Chen T, Li L, Tian J and Yu B: Erythropoietin alleviates hyperglycaemia-associated inflammation by regulating macrophage polarization via the JAK2/STAT3 signalling pathway. Mol Immunol 101: 221-228, 2018.
42. Yang X, Jia J, Yu Z, Duanmu Z, He H, Chen S and Qu C: Inhibition of JAK2/STAT3/SOCS3 signaling attenuates atherosclerosis in rabbit. BMC Cardiovasc Disord 20: 133, 2020.

43. Li CH, Xu LL, Zhao JX, Sun L, Yao ZQ, Deng XL, Liu R, Yang L, Xing R and Liu XY: CXCL16 upregulates RANKL expression in rheumatoid arthritis synovial fibroblasts through the JAK2/STAT3 and p38/MAPK signaling pathway. Inflamm Res 65: 193-202, 2016.

44. Liu Y, Holdbrooks AT, De Sarno P, Rowse AL, Yanagisawa LL, McFarland BC, Harrington LE, Raman C, Sabbaj S, Benveniste EN and Qin H: Therapeutic efficacy of suppressing the Jak/STAT pathway in multiple models of experimental autoimmune encephalomyelitis. J Immunol 192: 59-72, 2014.

45. Villarino AV, Kanno Y,Ferdinand JR and O'Shea JJ: Mechanisms of Jak/STAT signaling in immunity and disease. J Immunol 194: $21-27,2015$ 